\title{
New markers and models of premalignancy and the early development of gastric cancer
}

\section{Researchers identified a new specific marker for gastric stem cells}

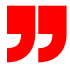

Clues to premalignancy and the early development of gastric cancer have been revealed in two new studies, highlighting the importance of gene-environment interactions during premalignancy and the role of stomach stem cells in gastric cancer.

In the first study published in Nature Genetics, researchers provided new insights into early events in gastric cancer by developing a new integrative mouse model of gastric premalignancy and generating premalignant organoids for further study. "Recent work from our group showed that, unlike other gastrointestinal cancers, inactivation of TP53 is an early event in gastric and oesophageal cancer, quite often occurring in premalignant lesions of patients who then progress to cancer. We, therefore, decided to develop a mouse model that integrated environmental risk factors, such as relevant dietary carcinogen exposure, with early $\operatorname{Trp} 53$ mutations to study gastric premalignancy," explains author Nilay Sethi.

Interestingly, certain premalignant phenotypes were not observed with either environmental exposures (nitrosamide) or TP53 alteration alone in various mouse models tested, but when these two factors were combined

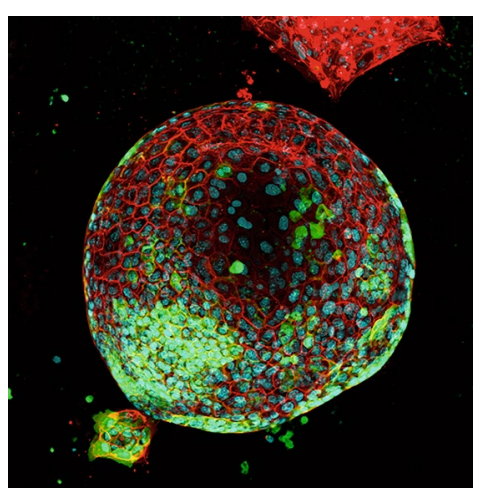

Mouse stomach organoid grown from a single $\mathrm{AQP5}^{+}$cell (green, AQP5; red, phalloid; cyan, nuclei). Image courtesy of N. Barker, A*STAR Institute of Medical Biology, Singapore. an increased number of dysplastic lesions were seen in the mice. Switching to a different model system, gastric organoids, the researchers observed a competitive advantage of TP53-altered gastric organoids when exposed to dietary carcinogens. Moreover, gastric organoids were generated from dysplastic lesions, enabling genomic, transcriptional and functional characterization of gastric premalignancy. Key features observed in these organoids included whole genome doubling, which often precedes aneuploidy and chromosomal instability, and induction of cell cycle regulators (most notably $C d k n 2 a$ ). Finally, co-deletion of Cdkn2a and Trp53 in dysplastic gastric organoids promoted cancer phenotypes as well as replication stress and activation of DNA damage response pathways.

In the second study published in Nature, researchers identified a new specific marker for gastric stem cells and modelled gastric cancer from these stem cells. "In the intestine, the LGR5-expresing cells serve as important sources of cancer following damage (mutation)," explains author Nick Barker. "When I moved to Singapore, the high prevalence of stomach cancers in Asia highlighted an unmet need to study the role of stem cells in tissue maintenance and cancer of the stomach," he adds. As LGR5 is commonly expressed across a range of different stem cell populations, Barker and colleagues wanted to identify a surface marker that was specific to stomach stem cells and that could facilitate the isolation of these cells from mice and humans.

Using comparative transcriptomic profiling of $\mathrm{LGR}^{+}$stem cell populations in the mouse gastrointestinal tract, the membrane protein aquaporin 5 (AQP5) was identified as a marker enriched in adult mouse pyloric stem cells, and then also in human pyloric stem cells. Using a newly generated mouse model, $\mathrm{AQP}^{+}$stem cells were confirmed as a source of WNT-driven, invasive gastric cancer in vivo. Additionally, $\mathrm{AQP}^{+}$gastric tumour cells could selectively initiate organoid growth in vitro and these organoids could be serially propagated in the absence of exogenous factors, indicative that this cell population contains potential cancer stem cells. Finally, AQP5 expression was assessed in 145 samples of human distal gastric cancer and was commonly expressed in primary intestinal and diffuse gastric cancer subtypes and their metastases.

Both sets of research established new experimental models of gastric cancer that could help improve our understanding of the early events in disease development and to better recapitulate human disease in a bid to find novel therapeutic targets. "One clear pathway forward is the development of preclinical models to test the utility of DNA damage response inhibitors in select genomic subtypes of gastric and oesophageal cancer," says Sethi. Barker is equally encouraged. "Understanding the mechanism of cancer formation and progression is critical to efforts to develop more selective, effective treatments," he notes. "Our ability to identify and purify these cancer stem cell-like populations from human tumours will allow us to directly evaluate their role in human cancer formation."

Katrina Ray

ORIGINAL ARTICLES Sethi, N. S. et al. Early TP53 alterations engage environmental exposures to promote gastric premalignancy in an integretative mouse model. Nat. Genet. 52, 219-230 (2020)| Tan, S. H. et al. AQP5 enriches for stem cells and cancer origins in the distal stomach. Nature $\mathbf{5 7 8}$, 437-443 (2020) 